\title{
Achieving Distributed Control through Model Checking
}

\author{
Susanne Graf ${ }^{1}$, Doron Peled ${ }^{2}$, and Sophie Quinton ${ }^{1}$ \\ 1 VERIMAG, Centre Equation, Avenue de Vignate, 38610 Gières, France \\ 2 Department of Computer Science, Bar Ilan University, Ramat Gan 52900, Israel
}

\begin{abstract}
We apply model checking of knowledge properties to the design of distributed controllers that enforce global constraints on concurrent systems. We calculate when processes can decide, autonomously, to take or block an action so that the global constraint will not be violated. When the separate processes cannot make this decision alone, it may be possible to temporarily coordinate several processes in order to achieve sufficient knowledge jointly and make combined decisions. Since the overhead induced by such coordinations is important, we strive to minimize their number, again using model checking. We show how this framework is applied to the design of controllers that guarantee a priority policy among transitions.
\end{abstract}

\section{Introduction}

Consider a concurrent system, where some global safety constraint, say of prioritizing transitions, needs to be imposed. A completely global coordinator can control this system and allow any of the maximal priority actions to progress in each state. However, the situation at hand is that of a distributed control 712]; controllers, one per process or set of processes, may restrict the execution of some of the transitions if their occurrence may violate the imposed constraint. Due to the distributed nature of the system, each controller has a limited view of the entire system. Each controller may keep some finite memory that is updated according to the history it can observe.

The knowledge of a process in any particular local state includes the properties that are common to all reachable (global) states containing it. There are several definitions for knowledge, depending on how much of the local history may be encoded in the local state. Knowledge was suggested as a tool for constructing a controller in 6 1. There, controlling a distributed system was achieved by first precalculating the knowledge of a process. Based on its precalculated knowledge, reflecting all the possible current situations of the other processes, a controller for a process may decide at runtime whether an action of the controlled process can be executed without violating the imposed constraint. Sometimes, however, the process knowledge is not sufficient. Then, the joint knowledge of several processes (also called distributed knowledge) may be monitored using fixed controllers for sets of processes. Unfortunately, this approach causes the loss of actual concurrency among the processes that are jointly monitored.

T. Touili, B. Cook, and P. Jackson (Eds.): CAV 2010, LNCS 6174, pp. 396-409, 2010.

(C) Springer-Verlag Berlin Heidelberg 2010 
Instead of permanent synchronizations via fixed process groups, we suggest in this paper a method for constructing distributed controllers that synchronize processes temporarily. We use model-checking techniques to precalculate a minimal set of synchronization points, where joint knowledge can be achieved during short coordination phases. An additional goal is synchronizing a minimal number of processes as rarely as possible. After each synchronization, the participating processes can again progress independently until a further synchronization is called for.

In [6], knowledge based controllability (termed Kripke observability) is studied as a basis for constructing a distributed controller. The problem there is somewhat different than ours: the goal is to make the system behave exactly according to a given regular language, while here we want to limit the possible choices in order to impose some given global invariant. There, if a transition is enabled by the controlled system but must be blocked according to the additional constraint, then at least one process knows that fact and is thus able to prevent its execution. This approach requires sufficient knowledge to allow any transition enabled according to a given regular specification. The construction in [1] is different: it requires that at least one process knows that the occurrence of some enabled transition preserves the correctness of the imposed constraint, hence supporting its execution. This approach preserves the correctness of the controller even when knowledge about other such transitions is limited, at the expense of restricting the choice of transitions.

The approach suggested here extends the knowledge based approach of [1]. We use a coordinator algorithm, such as the $\alpha$-core [5], which achieves temporary multiprocess coordinations using asynchronous message passing. Such coordinations can be used to achieve a precalculated joint knowledge, i.e., knowledge common to several processes. Such interactions are still expensive as they incur additional overhead. Therefore, an important part of our task is to minimize the number of interactions and the number of processes involved in such interactions.

\section{Preliminaries and Related Work}

Definition 1 (Distributed Transition systems). A distributed transition system $\mathcal{A}$ is a fivetuple $\langle\mathcal{P}, V, S, \iota, T\rangle$ :

$-\mathcal{P}$ is a finite set of processes.

$-V$ is a finite set of variables, each ranging over some finite domain. A process $p \in \mathcal{P}$ can access and change variables in $V_{p}$. Thus, $V=\cup_{p \in \mathcal{P}} V_{p}$. We do not require the sets $V_{p}$ to be disjoint.

- $S$ is the set of global states. Each state assigns a value to each variable in $V$ according to its domain.

$-\iota \in S$ is the initial state.

$-T$ is a finite set of transitions. A transition $\tau \in T$ consists of an enabling condition $e n_{\tau}$, which is a quantifier-free first order predicate, and a state transformation $f_{\tau}$. The transitions $T_{p} \subseteq T$ are associated with process $p$. Thus, $T=\cup_{p \in \mathcal{P}} T_{p}$. A transition $\tau$ may belong to more than one process and $P_{\tau}=\left\{p \mid \tau \in T_{p}\right\}$. Both enabling condition and transformation are over the variables $\cup_{p \in P_{\tau}} V_{p}$. 
Definition 2. A local state $\left.s\right|_{p}$ of a process $p \in \mathcal{P}$ is the restriction of a global state $s$ to the variables in $V_{p}$. Similarly, the joint local state $\left.s\right|_{P}$ of a set of processes $P \subseteq \mathcal{P}$ is the restriction of a global state to the variables in $\cup_{p \in P} V_{p}$.

For a set of states $S$ of a transition system, we denote the set of local states of process $p$ by $\left.S\right|_{p}$, and, respectively, the set of joint local states for set of processes $P \in \mathcal{P}$ by $\left.S\right|_{P}$. A transition $\tau$ is enabled in a state $s$ when $s=e n_{\tau}$ (i.e., $s$ satisfies $\left.e n_{\tau}\right)$. If $\tau$ is enabled in $s$ and $\tau$ is executed, a new state $s^{\prime}=f_{\tau}(s)$ is reached. We denote this by $s \stackrel{\tau}{\longrightarrow} s^{\prime}$.

Definition 3. An execution of a distributed system $\mathcal{A}$ is a maximal sequence $s_{0} s_{1} s_{2} \ldots$ such that $s_{0}=\iota$, and for each $i \geq 0, s_{i} \stackrel{\tau_{i}}{\longrightarrow} s_{i+1}$ for some $\tau_{i}$. A global state is called reachable if it appears in some execution sequence.

Definition 4. Given a system $\mathcal{A}$, a set of processes $P \subseteq \mathcal{P}$ knows in a state $s$ some property $\varphi$ over $V$, if $s^{\prime} \models \varphi$ for each reachable global state $s^{\prime}$ with $\left.s^{\prime}\right|_{P}=\left.s\right|_{P}$. We denote this by $s \models K_{P} \varphi$.

When $P$ is a singleton, we often write $p$ for the set $\{p\}$ as in $K_{p} \varphi$. It is easy to see that if $s=K_{P} \varphi$ and $\left.s\right|_{P}=\left.s^{\prime}\right|_{P}$ then also $s^{\prime} \models K_{P} \varphi$.

Definition 5. A finite state distributed disjunctive controller 712 for a system $\mathcal{A}=\langle\mathcal{P}, V, S, \iota, T\rangle$ is a set of automata $C_{p}=\left(L_{p}, \gamma_{p}, T_{p}^{o}, T_{p}^{c}, \rightarrow_{p}, E_{p}\right)$, one per process $p$ in $\mathcal{P}$, where:

- $L_{p}$ is the set of states of $C_{p}$, i.e., its finite memory.

- $\gamma_{p} \in L_{p}$ is the initial state of $C_{P}$.

- $T_{p}^{o}$ is the set of transitions observable by process $p$, satisfying $T_{p} \subseteq T_{p}^{o} \subseteq T$ : only the execution of transitions from $T_{p}^{o}$ can change the state of $C_{p}$.

$-T_{p}^{c}$ is the set of controllable transitions, where $T_{p}^{c} \subseteq T_{p}$. We require consistency between processes regarding controllability: if $\tau$ is involved with several processes, then it is either controllable by all of them or by none of them.

$-\rightarrow_{p}: L_{p} \times T_{p} \mapsto L_{p}$ is the transition function of $C_{p}$.

- $E_{p}:\left.S\right|_{p} \times L_{p} \mapsto 2^{T_{p}^{c}}$ is the support function, which in each local state returns the set of controlled transitions of process $p$ that $C_{p}$ supports (i.e., allows to proceed, when enabled).

A controller is designed to impose some constraint $\psi \subseteq S \times T$ on a given system $\mathcal{A}$, while not introducing any new deadlock.

Definition 6. A controlled execution of a distributed system $\mathcal{A}$ with controllers $C_{p}$ for $p \in \mathcal{P}$ is defined over a set of controlled states $G \subseteq S \times \Pi_{p \in \mathcal{P}} L_{p}$. Each controlled state $g \in G$ contains some global state $s \in S$, and a state $\rho_{p} \in L_{p}$ for each controller $C_{p}$. An execution $g_{0} g_{1} g_{2} \ldots$ is a maximal sequence of controlled states, satisfying that $g_{0}$ is the controlled state containing the initial states $\iota$ of $\mathcal{A}$ and $\gamma_{p}$ for each $C_{p}$. Furthermore, for each adjacent pair of controlled states $g_{i}$ and $g_{i+1}$ there exists a transition $\tau$ such that the following holds: 
1. $s \stackrel{\tau}{\longrightarrow} s^{\prime}$ - where $s \in S$ is the state component of the controlled state $g_{i}$ and $s^{\prime} \in S$ the one of $g_{i+1}$.

2. $\tau \in T_{p} \backslash T_{p}^{c} \cup E_{p}\left(\left.s\right|_{p}, \rho_{p}\right)$ for at least one process $p$; that is, either $\tau$ is uncontrollable by $p$ or $p$ supports $\tau$ in its current local state and given the state of its controller $C_{p}$.

3. For the states $\rho_{i}$ and $\rho_{i+1}$ of controller $C_{p}$ of $g_{i}$ and $g_{i+1}$, respectively, if $\tau \in T_{p}^{o}$, then $\rho_{i} \stackrel{\tau}{\longrightarrow} p \rho_{i+1}$. Otherwise, $\rho_{i}=\rho_{i+1}$. That is, $C_{p}$ changes its internal state when an observable transition occurs.

We denote by $\mathcal{A}_{c}$ the transformation of $\mathcal{A}$ that includes both $\mathcal{A}$ and its controllers.

Definition 7. A controller for $\mathcal{A}$ achieves a goal $\psi \subseteq S \times T$ if each transition $s \stackrel{\tau}{\longrightarrow} s^{\prime}$ (as in bullet 1. of Definition [6) satisfies that $(s, \tau) \in \psi$.

Note that the goal of the controller is to satisfy an invariant that is not just over the states (of the original system $\mathcal{A}$ ), but may also include the immediate transition out of that state. When no constraints on the transitions are imposed, we can use the simpler case where $\psi \subseteq S$.

The definition of a controller allows the use of some finite memory that is updated with the execution of observable transitions. This can be useful, e.g., when constructing a controller based on knowledge with perfect recall [1]. However, a controller based on simple knowledge, as in Definition 4 . does not have to exercise this capability, and $L_{p}$ can thus consist of a single state. As in [1, we fix as a running example a particular property that we want to synthesize: that of enforcing some priority policy on the distributed system.

Definition 8 (Priority policy). A priority policy $\operatorname{Pr}=(T, \ll)$ for a system $\mathcal{A}$ is defined as a partial order relation $\ll$ on the set of transitions $T$.

Among the transitions enabled in state $s$, we can identify those with maximal priority, i.e., enabled transitions such that for any other transition $\tau^{\prime}$ enabled in $s$, either $\tau^{\prime} \ll \tau$ or $\tau$ and $\tau^{\prime}$ are incomparable. Let $\max _{\tau}$ be a predicate that holds in a state $s$, i.e., $s \models \max _{\tau}$, when the transition $\tau$ has a maximal priority among the transitions enabled in $s$.

Definition 9. A prioritized execution of a system $\mathcal{A}$ according to a given priority policy $\operatorname{Pr}$ satisfies, in addition to the conditions of Definition 3 , that when $s_{i} \stackrel{\tau_{i}}{\longrightarrow} s_{i+1}$, then also $s_{i}=\max _{\tau_{i}}$.

The goal is then to construct a distributed controller for $\mathcal{A}$ such that, when running $\mathcal{A}$ together with its controller, only correctly prioritized executions occur. To prevent the situation where in some state an uncontrollable transition has lower priority than another enabled transition, we impose the restriction that uncontrollable transitions always have maximal priority.

Definition 10. For each local state $\left.s\right|_{p}$ of process $p$, define the following properties $k_{i}^{p}$ based on the knowledge of $p$ in that state. 
$-k_{1}^{p}=\bigvee_{\tau \in T_{p}} K_{p} \max _{\tau}$ : process $p$ can identify a transition $\tau$ such that it knows that $\tau$ is enabled with maximal priority.

- $k_{2}^{p}=\neg k_{1}^{p} \wedge K_{p} \bigvee_{q \neq p} k_{1}^{q}$ : process $p$ does not know whether it has a transition with maximal priority, but in all the global states $s^{\prime}$ with $\left.s^{\prime}\right|_{p}=\left.s\right|_{p}$ some other process $q$ is in a local state where $k_{1}^{q}$ holds. This allows $p$ to remain inactive without risk of introducing a deadlock.

- $k_{3}^{p}=\neg k_{1}^{p} \wedge \neg k_{2}^{p}: p$ does not know whether or not there is a supported transition.

$k_{1}^{p}$ can be extended to sets of processes: $k_{1}^{P}=\bigvee_{\tau \in \cup_{p \in P} T_{p}} K_{P} \max _{\tau}$.

Note that $k_{1}^{p} \vee k_{2}^{p} \vee k_{3}^{p} \equiv$ true. When the constraint $\psi$ imposed by the controller is different from the priority policy, the formula $k_{1}^{p}$ needs to be changed accordingly; instead of $\max _{\tau}$, it must reflect the property that executing $\tau$ does not invalidate $\psi$. If $\psi$ is a state property $(\psi \subseteq S)$, then $\max _{\tau}$ can be replaced by the state predicate $w p_{\tau}(\psi)$ (for "weakest precondition"), which reflects the state property that holds when $\tau$ is enabled and $\psi$ holds after its execution.

The construction in [1] checks whether $\bigvee_{p \in \mathcal{P}} k_{1}^{p}$ holds in all reachable states of the original system that are not deadlock (or termination). If so, it is sufficient that each process supports a transition when it knows that it is maximal in order to enforce the additional constraint $\psi$ (in that case, priority) without introducing any additional deadlock. When this check fails, it was suggested to monitor and control several processes together, or to use the more expensive knowledge of perfect recall (or to use both).

\section{A Synchronization Based Approach}

In this paper, we suggest a new solution to the distributed control problem, which consists of synthesizing distributed controllers that allow processes to temporarily synchronize in order to obtain joint knowledge in those (local) states in which it is needed. The synchronization is achieved by using an algorithm like $\alpha$-core [5]. This algorithm allows processes to notify, using asynchronous message passing, a set of coordinators about their wish to be involved in a joint action. We treat the synchronizations provided by the $\alpha$-core, or any similar algorithm, as transitions that are joint between several participating processes. At a lower level, such synchronizations are achieved using asynchronous message passing. We assume that the correctness of the algorithm guarantees the atomic-like behavior of such coordinations, allowing us to reason at this level of abstraction.

A joint local state $\left.s\right|_{P}$ satisfying $k_{1}^{P}$ indicates that the set of processes $P$ know how to act in this state by selecting some transition with maximal priority. Our construction calculates, using model checking for knowledge properties, which synchronizations are actually needed.

An exact check for the existence of a global (completely synchronized) controller can be based on game theory. Accordingly, one may present the problem as implementing a strategy for the following two player game. One player, the environment, can always choose between the enabled uncontrollable transitions, 
while the other player can choose between the enabled controllable ones. The goal of the controller is that the property $\psi$ is satisfied by the jointly selected execution. This can be solved using algorithms based on safety games 9 .

Our algorithm first calculates the local states and joint local states (synchronizations) providing sufficient knowledge to guarantee that in every global state at least one process supports some transition. We refer to this set of (joint) local states as the knowledge table $\Delta$ for $\mathcal{A}$. We use it to transform the system into a controlled system by implementing support to transitions: when the knowledge is not available locally, we add temporary synchronization between processes, according to the entries of the knowledge table. Finally, we propose to obtain a more efficient controller by minimizing the set of coordinations.

\subsection{A Set of Synchronizations Providing Sufficient Knowledge}

First, we calculate the required knowledge table $\Delta$. The construction of $\Delta$ is performed iteratively, starting with local states, then pairs of local states, triples etc. At each stage of the construction, $\Delta$ contains a set of (joint) local states $\left.s\right|_{P}$ satisfying $k_{1}^{P}$.

Definition 11. A set of (joint) local states $\Delta$ is an invariant of a system if each non-deadlock state of the system contains at least one (joint) local state from $\Delta$.

The first iteration includes in $\Delta$, for all $p \in \mathcal{P}$, the singleton local states satisfying $k_{1}^{p}$, i.e. states in which progress of $p$ is guaranteed. With each such local state $\left.s\right|_{p}$ we associate the actual transitions $\tau$ that make $k_{1}^{p}$ hold.

If $\Delta$ is not an invariant, we first calculate for each local state not satisfying $k_{1}^{p}$ whether it satisfies $k_{2}^{p}$. Let $U_{p}$ be the set of local states of process $p$ satisfying $\neg\left(k_{1}^{p} \vee k_{2}^{p}\right)$. Now, in a second iteration, we add to $\Delta$ pairs $\left(s_{p}, s_{q}\right) \in U_{p} \times U_{q}$ for $p \neq q$ if there exists a reachable state $s$ such that $\left.s\right|_{p}=s_{p}$ and $\left.s\right|_{q}=s_{q}$, and furthermore $s \models k_{1}^{\{p, q\}}$. Again, we associate with that entry of the table $\Delta$ the transitions $\tau$ that witness the satisfaction of $k_{1}^{\{p, q\}}$ for that entry. The second iteration terminates as soon as $\Delta$ is an invariant or if all such pairs of local states have been classified. In a third iteration, we consider triples of local states from $U_{p} \times U_{q} \times U_{r}$ such that no subtuple is in $\Delta$, and so forth.

\subsection{A Distributed Controller Imposing the Global Property}

We transform now the system $\mathcal{A}$ into a controlled transition system $\mathcal{A}_{c}$ allowing only prioritized executions. We implement $\mathcal{A}_{c}$ using a set of coordinators realizing the required synchronization of $\Delta$ by an algorithm such as the $\alpha$-core.

We want to achieve the joint local knowledge promised by the precalculation of $\Delta$ using synchronizations amongst the processes involved. Our construction guarantees that each time the transition associated with a tuple $\left(\left.\left.s\right|_{p_{1}} \ldots s\right|_{p_{k}}\right)$ from $\Delta$ is executed from a state that includes these local components, the property $\psi$ we want to impose is preserved. We transform the system $\mathcal{A}$ such that only transitions associated with entries in $\Delta$ can be executed. 
If a transition $\tau$ is associated with a singleton element $\left.s\right|_{p}$ in $\Delta$, then the controller for $p$, at the local state $\left.s\right|_{p}$, supports $\tau$. Otherwise, $\tau$ is associated with a tuple of local states in $\Delta$; when reaching any of these local states, the corresponding processes $p_{1} \ldots p_{k}$ try to achieve a synchronization, which consequently allows $\tau$ to execute. This is done according to the protocol of the synchronization algorithm that is used. Upon reaching the synchronization, the associated transition $\tau$ is then supported by any of its participating processes. Formally, for each transition $\tau$ associated with a tuple of local states $\left(\left.\left.s\right|_{p_{1}} \ldots s\right|_{p_{k}}\right)$, we execute a transition, enabled exactly in the joint local state with the above components, and performing the original transformation of $\tau$.

\subsection{Minimizing the Number of Coordinators}

It is wasteful to include a coordination for each joint local state involving at least two processes in $\Delta$. We now show how to minimize the number of coordinators for pairs of the form $\left(\left.s\right|_{p},\left.r\right|_{q}\right)$ in $\Delta$. The general version of this method for larger tuples is analogous. We denote by $\Delta_{p, q}$ the set of pairs of $\Delta$ made of a local state from process $p$ and one from process $q$.

A naive implementation may use a coordination for every pair in $\Delta$. Nevertheless, the large number of messages needed to implement coordination by an algorithm like $\alpha$-core suggests that we minimize the number of coordinations. A completely opposite extreme would be to use a unique coordination between processes $p$ and $q$. Accordingly, when process $p$ identifies that it may have a $q$ partner in $\Delta_{p, q}$, then coordination starts. When coordination succeeds, the joint event checks whether the local states of $p$ and $q$ actually appear in $\Delta_{p, q}$. If they do, it provides the appropriate behavior; otherwise, the coordination is abandoned. In this way, many (expensive) coordinations may be made just to be abandoned, not even guaranteeing eventual progress.

Consider now a set of pairs $\Gamma \subseteq \Delta_{p, q}$ such that if $(s, r),\left(s^{\prime}, r^{\prime}\right) \in \Gamma$, then $\left(s, r^{\prime}\right),\left(s^{\prime}, r\right) \in \Gamma$ ( $s$ and $s^{\prime}$ do not have to be disjoint, and neither do $r$ and $\left.r^{\prime}\right)$. This means that $\Gamma$ is a complete bipartite subgraph of $\Delta_{p, q}$. It is sufficient to generate one coordination for all the pairs in $\Gamma$. Upon success of the coordination, the precalculated table $\Delta_{p, q}$ will be consulted about which transition to allow, depending on the components $\left.s\right|_{p}$ and $\left.s\right|_{q}$. Thus, according to this strategy, a sufficient number of interactions is formed by finding a covering partition $\Gamma_{1}, \ldots, \Gamma_{m}$ of complete bipartite subgraphs of $\Delta_{p, q}$. That is, each pair $\left(\left.s\right|_{p},\left.r\right|_{q}\right) \in$ $\Delta_{p, q}$ must be in some set $\Gamma_{i}$. However, the minimization problem for such a partition turns out to be in NP-Complete.

Property 1. 4. Given a bipartite graph $G=(N, E)$ and a positive integer $K \leq|E|$, finding whether there exists a set of subsets $N_{1}, \ldots N_{k}$ for $k \leq K$ of complete bipartite subgraphs of $G$ such that each edge $(u, v)$ is in some $N_{i}$ is in NP-Complete.

We use the following notation: when $\Gamma$ is a set of pairs of local states, one from $p$ and one from $q$, we denote by $\left.\Gamma\right|_{p}$ and by $\left.\Gamma\right|_{q}$ the $p$ and the $q$ components in 
these pairs, respectively. We apply the following heuristics to calculate a (not necessarily minimal) set of complete bipartite subsets $\Gamma_{i} \subseteq \Delta_{p, q}$ covering $\Delta_{p, q}$. We start with a first partition $\Gamma_{1}^{0}, \ldots, \Gamma_{m_{0}}^{0}$, and refine it until we obtain a fixpoint $\Gamma_{1}^{k}, \ldots, \Gamma_{m_{k}}^{k}$. We decide to start with process $p$ if $\left.\left|\Delta_{p, q}\right|_{p}|<| \Delta_{p, q}\right|_{q} \mid$, i.e., the number of elements paired up in $\Delta_{p, q}$ is smaller for $p$ than for $q$. Otherwise, we symmetrically start with $q$. Let the elements of $\left.\Delta_{p, q}\right|_{p}$ be $x_{1}, \ldots x_{m_{0}}$, and $\Gamma_{i}^{0}$ be the pairs in $\Delta_{p, q}$ containing $x_{i}$. Now, we repeatedly alternate between the $q$ side and the $p$ side the following step: we check for each two sets $\Gamma_{i}^{l}$ and $\Gamma_{j}^{l}$ whether $\left.\Gamma_{i}^{l}\right|_{q}=\left.\Gamma_{j}^{l}\right|_{q}$. If it is the case, we combine them into a single set $\Gamma_{i}^{l} \cup \Gamma_{j}^{l}$. On even steps, we replace $q$ with $p$. This is done as long as we can unify new subsets in this way. The whole process is performed in time cubic in the size of $\Delta_{p, q}$.

Figure 1 shows the result for an example. The left-hand side represents the coordinators induced by $\Delta_{p, q}$ and the right-side the minimal set of coordinators. Each $\Gamma_{i}$ contains a single state of $q$. And indeed, if we start the procedure with $q$, the initial partition is already the solution.

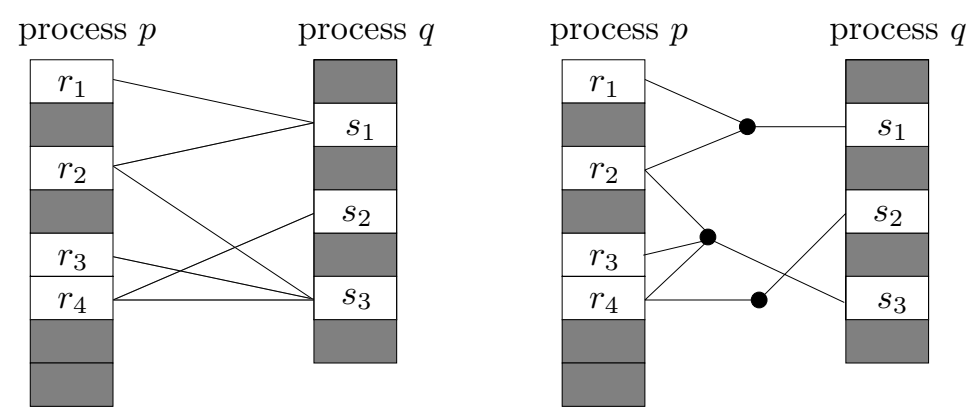

Fig. 1. Minimizing the number of coordinators

\section{Knowledge Based Controllers as a Practical Solution for the Distributed Control Problem}

We now show some connections between the classical controller synthesis problem (see, e.g., [7) and knowledge based control. We have provided a solution to the synthesis of distributed controllers, based on adding interactions between transitions in order to combine the knowledge of individual processes. In this section, we want to put the knowledge based solution in the context of the distributed control problem when adding interactions is not allowed. We first show an example where the local knowledge is not sufficient for controlling the system, but where blocking transitions - even when they are known to be maximal would allow controlling the system. This example shows that distributed controllers are more general than knowledge based controllers. However, there is no algorithm that guarantees constructing them: we show that even our limited problem (and running example) of controlling a system according to priorities is 
already undecidable. This advocates that the construction of knowledge based controllers, and furthermore, the use of additional synchronization, is a practical solution for the distributed control problem.

The knowledge approach to control in [6] requires that there is sufficient knowledge to allow any transition of the controlled system that does not violate the enforced property $\psi$. In [1, which we extend here, this requirement is relaxed; the knowledge must suffice to execute at least one enabled transition not violating $\psi$ when such a transition exists. In the more general case of distributed controller design, one may want to block some enabled transitions even if their execution does not immediately violate the enforced property. This is required to prevent the transformed system from later reaching deadlocked states, where the controlled system originally had a way to progress (thus, introducing new deadlocks).
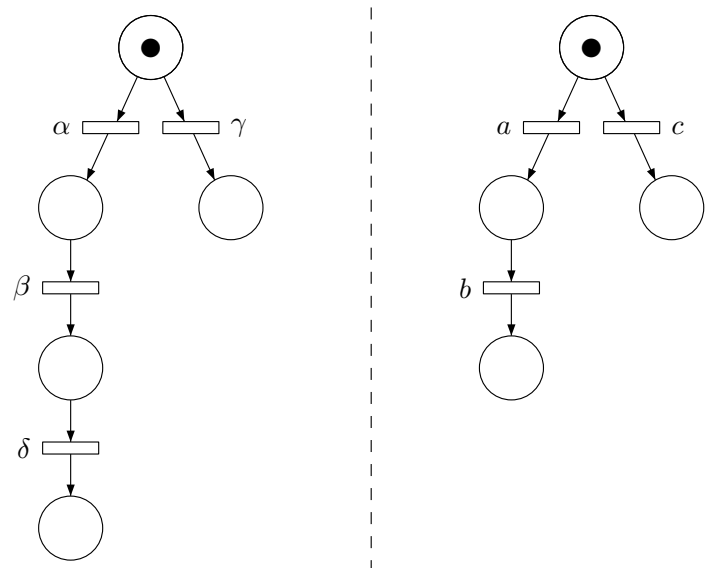

Fig. 2. A system with priorities $\delta \ll b \ll \beta$

Consider a concurrent system, as in Figure 2, with two processes $\pi_{l}$ (left) and $\pi_{r}$ (right), each one of them having initially a nondeterministic choice. The priorities in this system are $\delta \ll b \ll \beta$. Each process can observe only its own transitions. In the initial state, all four enabled transitions $\alpha, \gamma, a, c$ are unordered by priorities, and thus are all maximal. If $\alpha$ is fired and subsequently $a$ (or vice versa), we reach a global state where process $\pi_{r}$ does not have any enabled transition with maximal priority since $b \ll \beta$. Process $\pi_{l}$ does, and it can execute $\beta$. Thereafter, since $\delta \ll b$, process $\pi_{l}$ cannot execute $\delta$ and must wait for process $\pi_{r}$ to execute $b$. Now, with its limited observability, $\pi_{l}$ cannot distinguish between the situation before or after $b$ was executed by $\pi_{r}$. Thus $\pi_{l}$ lacks the capability, and the corresponding knowledge, of deciding whether to execute $\delta$. In this state, $\pi_{r}$ cannot distinguish between the situation before and after $\beta$ was executed, and cannot decide to execute $b$. Accordingly, the local knowledge of the processes in this example is not sufficient to construct a controller. In the initial state, both processes can progress freely, only to fall into a situation where they do not know locally when they can safely progress. 
When a controller is allowed to block transitions even when their execution does not immediately lead to violation of the property to be preserved, the situation can be recovered. In the example above, we may choose either to block $\alpha$ in favor of $\gamma$, or to block $a$ in favor of $c$. Blocking both $\alpha$ and $a$ is not necessary. This example also shows that there is no unique maximal solution to the control problem that blocks the smallest number of transitions. Note that an alternative solution to blocking $\alpha$ or $a$ can be achieved using a temporary interaction between the processes, as shown earlier in this paper.

It was shown in 10 , that the problem of synthesizing a distributed controller is, in general, undecidable. We show here that even when restricting the synthesis problem to priority policies, the problem remains undecidable. The proof for that is given below. Notice that when we have the flexibility of allowing additional coordination, as done in this paper, the problem, in the limit, becomes a sequential control problem, which is decidable.

Theorem 1. Constructing a distributed controller that enforces a priority policy is undecidable.

Proof. Following [10], the proof is by reduction from the post correspondence problem (PCP). In PCP, there is a finite set of pairs $\left\{\left(l_{1}, r_{1}\right), \ldots,\left(l_{n}, r_{n}\right)\right\}$, where the components $l_{i}, r_{i}$ are words over a common alphabet $\Sigma$, and one needs to decide whether one can concatenate separately a left word from the left components and a right word from the right components according to a mutual nonempty sequence of indexes $i_{1} i_{2} \ldots i_{k}$, such that $l_{i_{1}} l_{i_{2}} \ldots l_{i_{k}}=r_{i_{1}} r_{i_{2}} \ldots r_{i_{k}}$.

Let $i \in\{1 . . n\}, \hat{l}_{i}$ be the word $l_{i} i$, i.e., the $i^{\text {th }}$ left component concatenated with the index $i$. Similarly, let $\hat{r}_{i}$ be $r_{i} i$. We consider two regular languages: $L=\left(\hat{l}_{1}+\hat{l}_{2}+\ldots+\hat{l}_{k}\right)^{+}$and $R=\left(\hat{r}_{1}+\hat{r}_{2}+\ldots+\hat{r}_{k}\right)^{+}$. Now suppose a process $\pi_{p}$ executes according to the regular expression l.L.x.a.b+r.R.x.c.d. The choice of $\pi_{p}$ between $l$ and $r$ is uncontrollable. Suppose also that $\pi_{p}$ coordinates (through shared transitions) the alphabet letters from $\Sigma$ with a process $\pi_{q_{1}}$, and the indexes letters from $\Sigma$ with another process $\pi_{q_{2}}$. After that, $\pi_{q_{1}}$ and $\pi_{q_{2}}$ are allowed to interact with each other. Specifically, $\pi_{q_{2}}$ sends $\pi_{q_{1}}$ the sequences of indexes it has observed. Suppose that now $\pi_{q_{1}}$ has a nondeterministic choice between two transitions: $\alpha$ or $\beta$. The priorities are set as $b \ll \alpha \ll a$ and $d \ll \beta \ll c$. All other pairs of transitions are unordered according to $\ll$. If $\pi_{q_{1}}$ selects $\alpha$ and $r$ was executed, or $\pi_{q_{1}}$ selects $\beta$ and $l$ was executed, then there is no problem, as $\alpha$ is unordered with respect to $c$ and $d$, and also $\beta$ is unordered with respect to $a$ and $b$, respectively. Otherwise, there is no way to control the system so that it executes the sequence $a . \alpha . b$ or $c . \beta . d$ allowed by the priorities.

We show by contrapositive that if there is a controller, then the answer to the PCP problem is negative. Suppose the answer to the PCP problem is positive, i.e., some left and right words are identical and with the same indexes. Then process $\pi_{q_{1}}$ cannot make a decision: the information that $\pi_{q_{1}}$ observed and later received from $\pi_{q_{2}}$ is exactly the same in both cases for the mutual left and right word. Thus, $\pi_{q_{1}}$ cannot anticipate whether $c . d$ or $a . b$ will happen and cannot make a safe choice between $\alpha$ and $\beta$ accordingly. 
Conversely, if there is no controller, it means that $\pi_{q_{1}}$ cannot make a safe choice between $\alpha$ and $\beta$. This can only happen if $\pi_{q_{1}}$ and $\pi_{q_{2}}$ can observe exactly the same visible information for both an $l$ and an $r$ choice by $\pi_{p}$.

This means that deciding the existence of a controller for this system would solve the corresponding PCP problem. It is thus undecidable.

Note that in this proof we do not ensure a finite memory controller, even when one exists. Indeed, a finite controller may not exist. To see this, assume a PCP problem with one word $\{(a, a a)\}$. To check whether we have observed a left or a right word, we may just compare the number of $a$ 's that $p$ has observed with the number of indexes that $q$ has observed.

\section{Implementation and Experimental Results}

We have implemented a prototype for experimenting with this approach. In our tool, we use Petri nets to represent distributed transition systems.

This tool first builds the set of reachable states and the corresponding local knowledge of each process. Then, it checks whether local knowledge is sufficient to ensure correct distributed execution of the system under study. Let $\mathcal{U}$-states be global states in which all corresponding local states satisfy $\neg k_{1}^{p}$. The existence of a $\mathcal{U}$-state means that $\Delta$ is not an invariant without adding some tuples for synchronization. We allow simulating the system while counting the number of synchronizations and $\mathcal{U}$-states encountered during execution as a measurement of the amount of additional synchronization required.

The example that we used in our experiments is a variant of the dining philosophers where philosophers may arbitrarily take first either the fork that is on their left or right. In addition, a philosopher may hand over a fork to one of his neighbors when his second fork is not available and the neighbor is looking for a second fork as well. Such an exchange (labeled $e x$ ) is a way to avoid the wellknown deadlocks when all philosophers take first the fork on the same side. This example is partially represented by the Petri net of Figure 3 .

In our example, places (concerning philosopher $\beta$ ) are defined as follows:

- fork $^{i}$ : the $i$-th fork is on the table.

- 0 fork $_{\beta}$ (resp. 2 forks $_{\beta}$ ): philosopher $\beta$ has no fork (resp. 2 forks) in his hands.

- 1 fork $_{\beta}^{l}$ (resp. 1 fork $_{\beta}^{r}$ ): philosopher $\beta$ holds his left (resp. right) fork.

Transitions (concerning philosopher $\beta$ ) play the following role:

$-\operatorname{get}_{\beta}^{k l}$ (resp. $\operatorname{get}_{\beta}^{k r}$ ), $k=1,2$ : philosopher $\beta$ takes the fork on his left (resp. on his right). This is his $k$-th fork.

- eat-and-return ${ }_{\beta}$ : philosopher $\beta$ eats and puts both forks back on the table.

- ex $x_{\alpha, \beta}$ : philosopher $\alpha$ gives his right fork to philosopher $\beta$.

$-e x_{\beta, \alpha}$ : philosopher $\beta$ gives his left fork to philosopher $\alpha$.

Processes correspond to philosophers. The transitions defining a process $\beta$ have a $\beta$ in their name, including the four exchange transitions $e x_{\alpha, \beta}, e x_{\beta, \alpha}, e x_{\beta, \gamma}$ and $e x_{\gamma, \beta}$. 


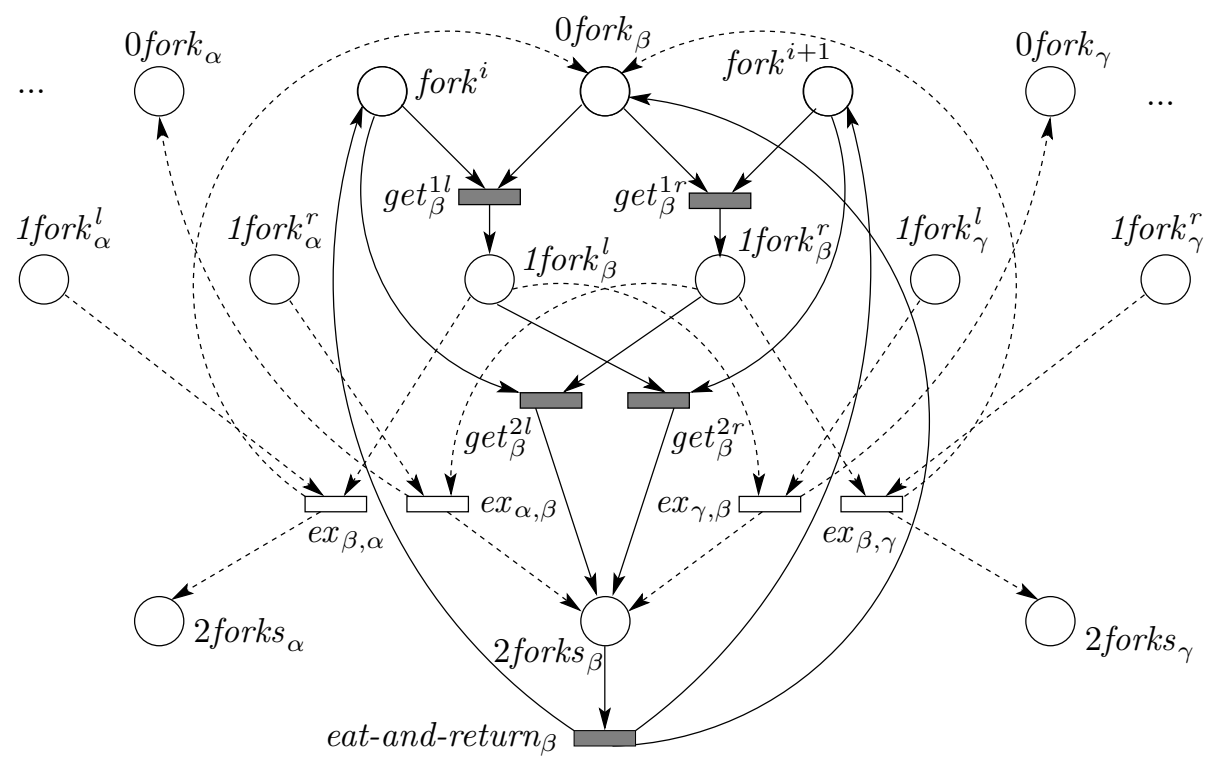

Fig. 3. A partial representation of the dining philosophers (philosopher $\beta$ )

In Figure 3 , transitions related only to philosopher $\beta$ are drawn with full lines. Transitions in dashed lines are shared between $\beta$ and one of his neighbors ( $\alpha$ on the left, $\gamma$ on the right).

Not controlling exchanges at all allows nonprogress cycles. To avoid them, we add priorities which allow exchange actions only when a blocking situation has been reached within some degree of locality.

First variant. We use a priority rule stating that an exchange between philosophers $\alpha$ and $\beta$ has lower priority than $\alpha$ or $\beta$ taking a fork. This leads to the following priorities for each $\alpha$ and $\beta$ such that $\alpha$ is $\beta$ s left neighbor:

$-e x_{\alpha, \beta} \ll g e t_{\alpha}^{2 l}$ : if $\alpha$ can pick up a left fork, he won't give his right fork to $\beta$.

$-e x_{\beta, \alpha} \ll g e t_{\beta}^{2 r}$ : symmetrically if $\beta$ can pick up a right fork.

In this variant, local knowledge is sufficient. Indeed, when a philosopher $\alpha$ and both his neighbors are blocked in a state where they all have a left (resp. a right) fork, then philosopher $\alpha$ has enough knowledge to support an exchange with his left (resp. right) neighbor. For any number of philosophers, there is no $\mathcal{U}$-state. Thus, no extra synchronization is needed.

Second variant. Now, to further reduce the number of exchanges, one may decide that philosopher $\beta$ may give his left fork to his left neighbor $\alpha$ only if (1) $\alpha$ is blocked (2) $\beta$ is blocked and (3) $\beta$ s right neighbor $\gamma$ is also blocked (the exchange of right forks is similar). This translates into adding the following priorities:

- ex $x_{\alpha, \beta} \ll g e t_{\delta}^{2 l}$, eat-and-return $\delta$ (with $\delta$ the left neighbor of philosopher $\alpha$ )

- $e x_{\beta, \alpha} \ll$ get $_{\gamma}^{2 r}$, eat-and-return $($ with $\gamma$ the right neighbor of philosopher $\beta$ ) 
Local knowledge alone cannot ensure here correct distributed execution. However, binary synchronizations are sufficient in this example to ensure that the system is always able to move on, for any number of philosophers.

In Table 1, we show results for the second variant with 6,8 and 10 philosophers. There are two $\mathcal{U}$-states which correspond to the situation where all philosophers hold their left fork, or they all hold their right fork. For computing the number of synchronizations, we used each time 100 runs of a length of 10,000 steps (i.e. transitions). Note that the number of exchange transitions is identical to the number of synchronizations.

Table 1. Results for 100 executions of 10,000 steps for the second variant

\begin{tabular}{|c|c|c|c|}
\hline philosophers & 6 & 8 & 10 \\
\hline reachable states & 729 & 6561 & 59049 \\
\hline synchronizations & 354 & 285 & 237 \\
\hline $\mathcal{U}$-states encountered & 253 & 149 & 100 \\
\hline
\end{tabular}

At the current stage, the minimization of the set of coordinators has not been implemented (we use one coordinator per synchronization pair in $\Delta$ ) and our tool handles only joint local states consisting of two states.

\section{Conclusion}

Imposing a global constraint upon a distributed system by blocking transitions is, in general, undecidable [108]. One practical approach for this problem was to use model checking of knowledge properties [1. If we allow additional synchronization, the problem becomes decidable: at the limit, everything becomes synchronized, although this, of course, is highly undesirable. The method presented in [1] provided a (disjunctive) controller. The problem with that approach is that in many cases the local knowledge of the separate processes does not suffice. A suggested remedy was to monitor several processes together, achieving this way an increased level of knowledge.

In the current work we look at the situation where we are allowed to coordinate between several processes, but only temporarily. First, we can calculate whether the constraint we want to impose is feasible, when all processes are combined together. This is done using game theory [9]. If this is the case, we check if we can control the system based on the local knowledge of processes or temporary interactions between processes. Of course, our goal is to minimize the number of interactions, and moreover, the number of processes involved in each interaction.

For achieving a distributed implementation, one can use a multiparty synchronization algorithm such as the $\alpha$-core algorithm [5]. Based on that, we presented an algorithm that uses model checking to calculate when synchronization between local states is needed. The synchronizing processes, successfully coordinating, are then able to use the knowledge table calculated by model checking, 
which dictates to them which transition can be executed. Some small corrections to the original presentation of the $\alpha$-core algorithm appear in [3] .

The framework suggested in this paper can be used as a distributed implementation for the Verimag BIP system [2] BIP is based on a clear separation between the behavior of atomic components and the interaction between such components, which is represented using (potentially hierarchical) connectors. Priorities offer a mechanism to enforce scheduling policies by filtering the set of interactions that can be fired. So far, implementing BIP systems in a distributed setting remains a challenging task.

\section{References}

1. Basu, A., Bensalem, S., Peled, D., Sifakis, J.: Priority scheduling of distributed systems based on model checking. In: Bouajjani, A., Maler, O. (eds.) CAV 2009. LNCS, vol. 5643, pp. 79-93. Springer, Heidelberg (2009)

2. Basu, A., Bozga, M., Sifakis, J.: Modeling heterogeneous real-time components in BIP. In: SEFM, pp. 3-12. IEEE Computer Society Press, Los Alamitos (2006)

3. Katz, G., Peled, D.: Code mutation in verification and automatic code generation. In: TACAS. LNCS. Springer, Heidelberg (to appear, 2010)

4. Orlin, J.B.: Contentment in graph theory: covering graphs with cliques (1977)

5. Pérez, J.A., Corchuelo, R., Toro, M.: An order-based algorithm for implementing multiparty synchronization. Concurrency - Practice and Experience 16(12), 1173-1206 (2004)

6. Rudie, K., Ricker, S.L.: Know means no: Incorporating knowledge into discreteevent control systems. Transactions on Automatic Control 45(9), 1656-1668 (2000)

7. Rudie, K., Wonham, W.M.: Think globally, act locally: decentralized supervisory control. Transactions on Automatic Control 37(11), 1692-1708 (1992)

8. Thistle, J.G.: Undecidability in decentralized supervision. System and Control Letters 54, 503-509 (2005)

9. Thomas, W.: On the synthesis of strategies in infinite games. In: Mayr, E.W., Puech, C. (eds.) STACS 1995. LNCS, vol. 900, pp. 1-13. Springer, Heidelberg (1995)

10. Tripakis, S.: Undecidable problems of decentralized observation and control on regular languages. Inf. Process. Lett. 90(1), 21-28 (2004)

11. van der Meyden, R.: Common knowledge and update in finite environments. Inf. Comput. 140(2), 115-157 (1998)

12. Yoo, T.-S., Lafortune, S.: A general architecture for decentralized supervisory control of discrete-event systems. Discrete Event Dynamic Systems 12(3), 335-377 (2002) 